\title{
The orphan nuclear receptor COUP-TFII is required for angiogenesis and heart development
}

\author{
Fred A. Pereira, ${ }^{1}$ Yuhong Qiu, ${ }^{1}$ Ge Zhou, Ming-Jer Tsai, ${ }^{2}$ and Sophia Y. Tsai ${ }^{2,3}$ \\ Department of Cell Biology, Baylor College of Medicine, Houston, Texas 77030 USA
}

\begin{abstract}
The embryonic expression of COUP-TFII, an orphan nuclear receptor, suggests that it may participate in mesenchymal-epithelial interactions required for organogenesis. Targeted deletion of the COUP-TFII gene results in embryonic lethality with defects in angiogenesis and heart development. COUP-TFII mutants are defective in remodeling the primitive capillary plexus into large and small microcapillaries. In the COUP-TFII mutant heart, the atria and sinus venosus fail to develop past the primitive tube stage. Reciprocal interactions between the endothelium and the mesenchyme in the vascular system and heart are essential for normal development of these systems. In fact, the expression of Angiopoietin-1, a proangiogenic soluble factor thought to mediate the mesenchymal-endothelial interactions during heart development and vascular remodeling, is down-regulated in COUP-TFII mutants. This down-regulation suggests that COUP-TFII may be required for bidirectional signaling between the endothelial and mesenchymal compartments essential for proper angiogenesis and heart development.
\end{abstract}

[Key Words: Angiogenesis; heart defects; COUP-TFII; nuclear receptors]

Received January 28, 1999; revised version accepted March 1, 1999.

The entire inner surface of blood vessels and the heart is lined with mesodermally derived endothelial cells. These specialized epithelial-like cells regulate essential vascular functions such as blood-tissue exchange, blood-tissue barriers, fibrinolysis and coagulation, and activation of the circulating immune system. Interestingly, both the vascular system and heart require cross-talk between endothelial cells and the surrounding mesenchymal cells for proper development (Dumont et al. 1994; Lee et al. 1995; Meyer and Birchmeier 1995; Suri et al. 1996). However, little is known about the underlying genetic and molecular determinants that control cardiac chamber formation (Fishman and Olson 1997) or blood vessel formation (Risau 1997). Vertebrate embryonic vascular system development involves two distinct processes (for reviews, see Risau and Flamme 1995; Folkman and D'Amore 1996; Hanahan 1997; Risau 1997). Vasculogenesis is the process by which mesodermal precursor cells commit to the endothelial lineage as angioblasts. These cells adhere and differentiate into endothelial tubes that are first organized into a primitive vascular plexus and then into larger blood vessels including the dorsal aorta. In contrast, angiogenesis involves the sprouting of new vessels from pre-existing vessels or splitting of larger vessels to form a microcapillary net-

\footnotetext{
${ }^{1,2}$ These authors contributed equally to this work.

${ }^{3}$ Corresponding author.

E-MAIL stsai@bcm.tmc.edu; FAX (713) 798-8227.
}

work (Risau 1997). Subsequently, vascular remodeling converts the primary capillary plexus into large and small vessels of the mature vasculature. The assembly of mature blood vessels, whether via vasculogenesis or angiogenesis, is dependent on bidirectional signaling between the endothelial cells and the surrounding mesenchymal cells (Folkman and D'Amore 1996). Many genes have been identified that regulate the processes of vasculogenesis, angiogenesis, or both. In addition, some genes have been shown to maintain the integrity of vessels by recruitment and formation of the periendothelial layer or by interactions between arteries and veins (Folkman and D'Amore 1996; Hanahan 1997; Risau 1997; Wang et al. 1998).

The Angiopoietin-1 (ANG1)-TIE2 signal transduction pathway involves a ligand-receptor tyrosine kinase (RTK) interaction important for development of both the vascular system and heart (Sato et al. 1995; Suri et al. 1996; Maisonpierre et al. 1997). ANG1 is a potent angiogenic factor that binds to and activates the endothelialspecific RTK, TIE2/TEK (Davis et al. 1996). ANG1 is expressed in the myocardium and in mesenchymal cells adjacent to developing endothelia of the intersegmental vessels, in the peritoneum and in the periocular mesenchyme (Davis et al. 1996). In the embryo, TIE2 is expressed in all endothelial cells (Yamaguchi et al. 1993). Ablation of either Ang1 or Tie2 results in profound vasculature and heart defects (Dumont et al. 1994; Sato et al. 1995; Suri et al. 1996). Ang1 mutants have an abnor- 
mal vasculature characterized by decreased branching and impaired recruitment of periendothelial smooth muscle and pericyte cells (Suri et al. 1996). In addition, these mutants show prominent endocardial and myocardial defects resulting in a reduced complexity of the ventricular endocardium and an almost collapsed atrial endothelial lining (Suri et al. 1996). Similar to mutants of its ligand ANG1, Tie2 mutants have deficiencies in angiogenesis and development of the heart (Dumont et al. 1994; Sato et al. 1995). ANG2, which antagonizes TIE2 signaling, is expressed in a distinct but overlapping pattern to that of ANG1, and ectopic overexpression results in similar defects in angiogenesis and heart development (Maisonpierre et al. 1997). Interestingly, enhanced TIE2 activity in humans results in venous malformations characterized by vasodilation and a lack of smooth muscle cells (Vikkula et al. 1996). Thus, any perturbation of the ANG1-TIE2 signal transduction pathway results in angiogenic defects in remodeling the primitive vascular plexus and heart defects due to inappropriate myocardial-endocardial interactions. Taken together, these studies demonstrate that multiple steps are involved in establishing the mature cardiac and vascular system and that normal development requires a balance of positive and negative factors (Folkman and D'Amore 1996).

The present studies reveal a novel effector that contributes to heart and blood vessel formation. Chicken ovalbumin upstream promoter-transcription factor II (COUP-TFII) is a member of the steroid/thyroid hormone receptor superfamily (for review, see Tsai and Tsai 1997). This nuclear receptor superfamily consists of a large group of ligand-activated transcription factors. No known ligands have been identified for more than half of the members, which have a well-defined putative ligandbinding domain $(\mathrm{LBD}$; for review, see Tsai and O'Malley 1994). Although COUP-TFs are well characterized biochemically, the precise physiological roles of COUP-TFs are largely undefined. The two mouse genes, COUP-TFI and COUP-TFII, are typical orphan receptors that share an exceptional degree of homology at the amino acid level $[98 \%$ identity in the DNA-binding domain (DBD) and $96 \%$ identity in the putative LBD; Qiu et al. 1994]. Furthermore, their expression patterns are distinct but overlap in many regions (Jonk et al. 1994a; Qiu et al. 1994; Pereira et al. 1995), suggesting that they may serve redundant functions. Sequence comparisons of the multiple COUP-TF homologs from invertebrates and vertebrates (Tsai and Tsai 1997) also revealed an exceptional evolutionary conservation, as exemplified by the $>89 \%$ amino acid identity of the fruit fly and human COUP-TF LBDs. This identity suggests a conservation of function. The expression patterns of vertebrate homologs are similar in the developing central nervous system, suggesting that they are also involved in neurogenesis (Fjose et al. 1993, 1995; Lutz et al. 1994; van der Wees et al. 1996). Indeed, COUP-TFI is required for axon guidance, arborization, and morphogenesis of the ninth cranial ganglion and nerve (Qiu et al. 1997), and the Drosophila homolog seven-up (Mlodzik et al. 1990) is required for photore- ceptor cell fate determination. Taken together, these results demonstrate clearly that COUP-TFI and its homologs are critical factors involved in neurogenesis.

In contrast, COUP-TFII is highly expressed in mesenchymal cells during organogenesis (Pereira et al. 1995; Tsai and Tsai 1997). This expression led us to hypothesize that COUP-TFII is required for mesenchymal-epithelial signaling during organogenesis. To assess the physiological function of COUP-TFII in vivo we now have disrupted COUP-TFII by targeted recombination in embryonic stem (ES) cells. Two-thirds of the heterozygote COUP-TFII mice die before weaning, and homozygous deletion of COUP-TFII is lethal around 10 days of gestation. Embryos are growth retarded, and have severe hemorrhage and edema by E9.5. Histological analyses revealed enlarged blood vessels, a lack of normal development of the atria and sinus venosus, and malformed cardinal veins. Immunological and molecular analyses of the vascular system show a decrease in the extent and complexity of the microvasculature in the head and spine regions, suggesting that angiogenesis and vascular remodeling are defective in COUP-TFII mutants. These defects are consistent with a loss of COUP-TFII function in the mesenchymal compartments of the head, spine, and heart. Analyses of multiple ligand-RTK pathways known to regulate primitive vascular development revealed that Ang1 is down-regulated in COUP-TFII mutants. Perturbations of the ANG1-TIE2 pathway may contribute to the heart and vasculature defects observed in COUP-TFII mutants. These results suggest that COUP-TFII may modulate embryonic heart and vasculature patterning via mesenchymal-endothelial signaling.

\section{Results}

\section{COUP-TFII expression during mouse development}

The early expression of COUP-TFII is consistent with a role in mesenchymal-endothelial interactions during embryonic heart and vascular development. COUP-TFII was detected in the visceral mesoderm and differentiating myocardium of the sinus venosus as the heart undergoes a rightward looping and in the mesoderm surrounding the umbilical veins (Fig. 1A). The inflow tract is then shifted dorsally and superiorly, and the atrial segment and a part of the sinus venosus are taken up into the pericardial cavity (Ulrich 1995). By E9, COUP-TFII expression is up-regulated during expansion of the common atrium and enlargement of the bilaterally symmetrical sinus venosus (Fig. 1B). COUP-TFII transcripts are also found in the mesenchyme of the developing umbilical veins, somite, otocyst, eye, and hindbrain. Thus, the early expression of COUP-TFII is consistent with a role in patterning the sinus venosus, the common atrium, and parts of the vascular and organ systems, which are dependent on mesenchymal-epithelial interactions.

During mid-gestation, COUP-TFII is expressed in the mesenchymal cells of the frontonasal process, sense or- 


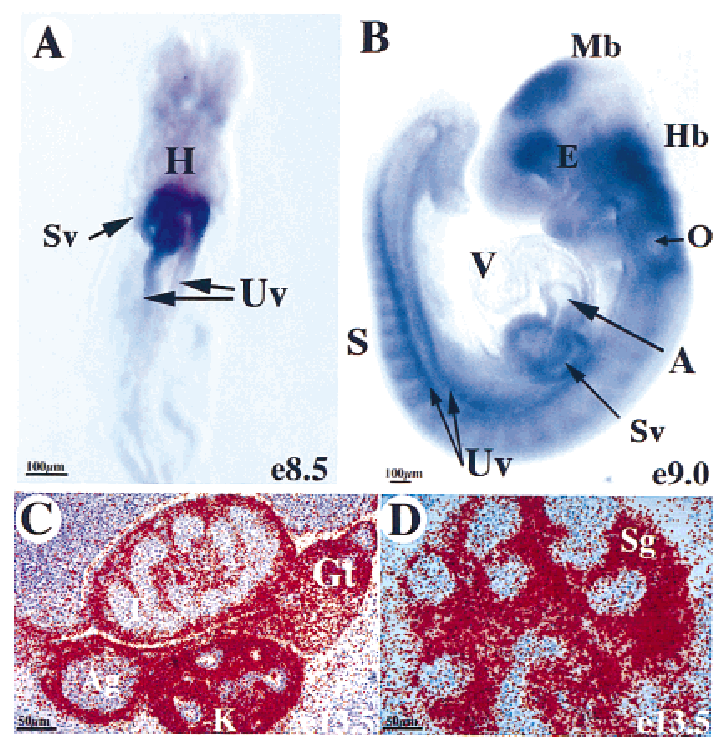

Figure 1. COUP-TFII expression. (A) Whole-mount in situ hybridization of an E8.5 embryo showing COUP-TFII expression in the expanding sinus venosus $(\mathrm{Sv})$ and in the elongating umbilical veins $(\mathrm{Uv})$ (ventral view, positive signal is bluish purple color). (H) Heart. (B) At E9, (E9.0), COUP-TFII is detected in the developing hindbrain $(\mathrm{Hb})$, otocyst $(\mathrm{O})$, the periocular mesenchyme and optic stalk (E), and expression in the anterior midbrain $(\mathrm{Mb})$ is restricted to the neuroectoderm. Expression is also seen in the expanding common atrium (A), in the bilaterally symmetrical sinus venosus, and caudally in the developing umbilical veins. Expression in the somite (S), which was first detected at E8.5 (not shown), is now readily detected in all somites. (V) Ventricle. (C) COUP-TFII is expressed in the mesenchyme of the developing testis $(\mathrm{T})$, genital tubercle $(\mathrm{Gt})$, kidney $(\mathrm{K})$, and cortex of the adrenal gland $(\mathrm{Ag})$ at E13.5. Shown is a sagittal view of a double exposure with the positive signal in red. $(D)$ COUP-TFII expression at E13.5 is restricted to the mesenchyme throughout salivary gland $(\mathrm{Sg})$ development.

gans, respiratory, digestive, and urogenital systems (Pereira et al. 1995). High magnification of the urogenital region at E13.5 shows expression in the mesenchyme of the kidney, genital tubercle, gonad, cortex of the adrenal gland (Fig. 1C), and in the salivary gland (Fig. 1D). Expression levels decline dramatically after completion of organogenesis and reach basal levels in the adult. In general, COUP-TFII expression is detected in the mesenchyme but not in the terminally differentiated epithelium. Collectively, these results support the hypothesis that COUP-TFII may regulate signals required for induction of epithelial cell differentiation and may play different roles at specific stages of embryonic development through a common mechanism.

\section{Targeted disruption of the COUP-TFII gene}

A mouse $129 \mathrm{~Sv}$ genomic clone spanning the entire COUP-TFII gene was used to generate the targeting construct. The COUP-TFII amino terminus and DBD are contained in the first exon, whereas the LBD is split into two exons (Qiu et al. 1995). The targeting vector contained $2.2 \mathrm{~kb}$ of $5^{\prime}$ homologous sequence and $3.0 \mathrm{~kb}$ of $3^{\prime}$ homologous sequence flanking the $P G K n e o b p A$ neomycin-resistance cassette (Fig. 2A). The $n e o^{\mathrm{r}}$ gene was inserted in the same transcriptional direction as COUPTFII. On correct recombination, a 3.2-kb fragment (StuISalI) containing most of exon 1 and the entire exon 2 was deleted and replaced with $P G K n e o b p A$ for positive selection. Thus, most of the amino terminus, the entire DBD and two-thirds of the LBD were deleted ensuring that no partial COUP-TFII protein with a dominantnegative function was generated. To select against random insertions, a negative selection with the herpes simplex virus thymidine kinase gene was included in the targeting vector. The targeting vector was linearized and electroporated into $\mathrm{AB} 1$ or $\mathrm{AB} 2.2 \mathrm{ES}$ cells, which were subjected to positive (G418) and negative (FIAU) selections. Multiple COUP-TFII heterozygous ES cell clones were obtained from each electroporation, and proper recombination was confirmed by Southern analyses with probes $5^{\prime}$ or $3^{\prime}$ to the targeting vector (Fig. 2B).

COUP-TFII heterozygous ES cell lines were injected into C57BL/ 6 blastocysts, several male chimeras with a high percentage of agouti coat color were generated, and germ-line transmission was obtained from two independent ES clones. Heterozygotes were one-third smaller than wild-type littermates by 5 days of birth (data not shown) and approximately two-thirds were lost before weaning at 21 days postpartum (see Table 1). Thus, loss of a single copy of COUP-TFII results in heterozygote insufficiency and poor postnatal viability. Heterozygotes that survived were fertile and were intercrossed to generate homozygotes. In an attempt to improve the viability, COUP-TFII heterozygotes (129Sv $\times$ C57BL/6) were crossed into the outbred ICR background or into pure inbred 129Sv or C57BL/ 6 backgrounds. These three independent genetic backgrounds did not improve the viability of heterozygotes or homozygotes. The following phenotypic observations occurred with $100 \%$ penetrance in the two independent mouse lines and in multiple genetic backgrounds.

\section{COUP-TFII gene function is essential for embryogenesis}

To define the physiological consequences of deleting COUP-TFII in vivo, $\mathrm{F}_{1}$ heterozygotes were intercrossed to generate homozygotes. No homozygotes were recovered at weaning during an initial analysis of $132 \mathrm{~F}_{2}$ progeny (Table 1). Further analysis of heterozygous intercrossings revealed no COUP-TFII homozygotes at birth indicating that a homozygous mutation at the COUPTFII locus results in embryonic lethality. At E9.5, Mendelian ratios of wild-type, heterozygotes, and homozygotes were readily detected (Table 1) by Southern analyses (not shown) and PCR (Fig. 2C). However, homozygotes were phenotypically abnormal, with hemorrhaging in the frontonasal process (Fig. $3 \mathrm{~B}$ and $\mathrm{C}$ ) and edematous cysts on the brain (arrows in Fig. 3C; see also Fig. 6B, below). At E10-E10.5, there was a decreased 
Figure 2. Targeted disruption of the COUP-TFII locus. (A) Targeting vector construction. (Shaded boxes I, II, and III) COUP-TFII-coding regions; (open box) $5^{\prime}$ untranslated region; (arrows in boxes) direction of transcription. (TK) Thymidine kinase gene; $\left(\right.$ neo $\left.^{\mathrm{r}}\right)$ neomycin-resistance gene. (R) EcoRI; (St) StuI; (Sl) SalI; (X) XbaI; (H) HindIII; (Sp) SpeI; (Xh) XhoI. (B) Southern blot analysis. (WT) Wild-type allele; (KO) recombined allele. Of the 680 colonies screened, 17 positive clones were obtained for a targeting efficiency of $2.5 \%$. To ensure proper recombination had occurred at both the $5^{\prime}$ and $3^{\prime}$ ends of the neor cassette, Southern analysis was performed with probes $5^{\prime}$ and $3^{\prime}$ of the targeting vector. The $5^{\prime}$ probe was a $0.8-\mathrm{kb}$ EcoRI fragment with $X b a \mathrm{I}$ as the diagnostic enzyme as the neo ${ }^{\mathrm{r}}$ cassette (see $A$ ) introduced a new $X b a I$ site to the locus. The expected $10-\mathrm{kb}$ wild-type and $8-\mathrm{kb}$ mutant fragments were generated (left). The 3' probe was a 1-kb EcoRV-NsiI fragment downstream of the $3^{\prime}$ homologous sequence and SpeI was the diagnostic enzyme that produced a $12-\mathrm{kb}$ wild-type and a 6-kb mutant band (right). Reprobing the blots with the $n e o^{\mathrm{r}}$ gene revealed no other insertions in the genome /data not shown). Therefore, correct recombination was obtained at the COUP-TFII locus to inactivate the gene. (C) PCR genotyping showing wild-type $(+\mid+)$, heterozygote $(+)$ -1 , and mutant $(-/-)$ embryos from the two independent (E5 and H7) COUP-TFII targeted lines. (D) RT-PCR analysis of transcripts from E9.5 embryos. There are no products in the absence of reverse transcriptase (RT) indicating that the bands are products of reverse transcription, and there are no wild-type COUP-TFII transcripts in the mutants (-/-). (KO) An amplified product from the 5 ' end of the locus and the neor gene.

number of homozygotes (Table 1). Mutant embryos were dramatically bloody as the brain vesicles were often filled with blood (Fig. 3E) or the hearts were hemorrhagic (Fig. 3F). These partially resorbed embryos never had more than 29 somites as compared with the typical wildtype embryo at this stage with 30-35 somites (Fig. 3D). Thus, COUP-TFII mutants die around E10 possibly as a result of hemorrhage and edema in the brain and heart. Surprisingly, the number of heterozygotes was also below the expected Mendelian ratio and $~ 5 \%$ of heterozygotes isolated at E9.5 presented with a similar hemorrhagic phenotype as homozygotes (data not shown). No homozygotes were ever detected at E11.5 (data not shown). To ensure no COUP-TFII transcripts were generated in homozygotes, E9.5 embryos genotyped to be mutants were analyzed by in situ hybridization and found to have no signals above background levels (data not shown). In addition, by use of RT-PCR of E9.5 RNA, no COUP-TFII-specific wild-type transcripts amplified from exon 2, which should be deleted, were detected in homozygotes (Fig. 2D). In the absence of the reverse transcriptase enzyme, no amplified fragments were detected ensuring that COUP-TFII was indeed mutated. These data suggest that the function of COUP-TFII is essential for embryogenesis and even in the heterozygous state, some embryos do not survive gestation. Thus, analysis of the role of COUP-TFII in mesenchymal-epithelial interactions during organogenesis will require tissue-specific or conditional knockout strategies.

Table 1. Genotype of COUP-TFII progeny from heterozygous matings

\begin{tabular}{lcccc}
\hline Age & Total & $+/+$ & $+/-$ & $-/-$ \\
\hline Adults & 132 & 80 & 52 & 0 \\
& & $(61 \%)$ & $(39 \%)$ & $(0)$ \\
E9.0-9.5 & 232 & 56 & 121 & $55^{\mathrm{a}}$ \\
& & $(24 \%)$ & $(52 \%)$ & $(24 \%)$ \\
E10.0-10.5 & \multirow{2}{*}{135} & 43 & 66 & $26^{\mathrm{b}}$ \\
& & $(32 \%)$ & $(49 \%)$ & $(19 \%)$ \\
\hline
\end{tabular}

$\overline{\text { Each number represents an individual animal, with percentages }}$ in brackets.

aAbnormal.

${ }^{\mathrm{b}}$ Resorbing. 


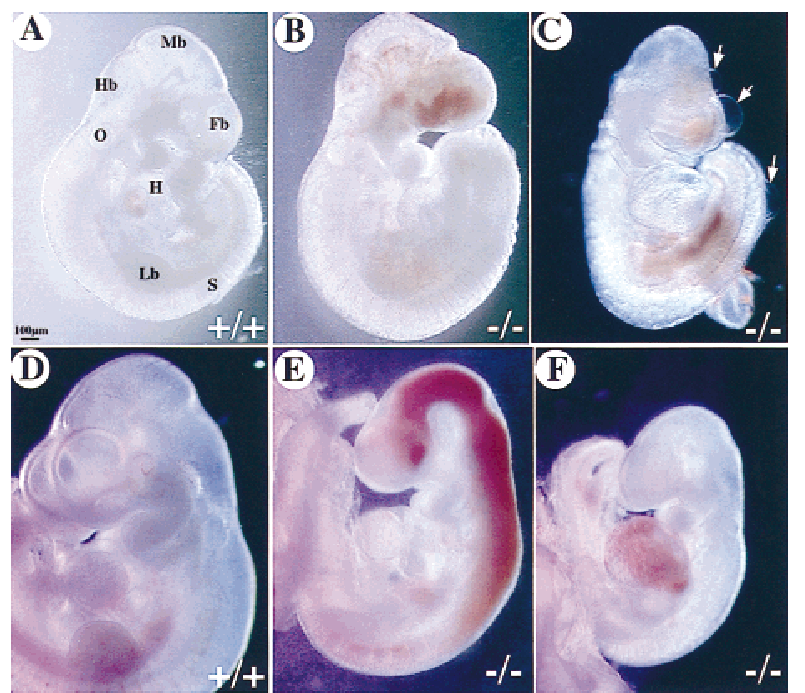

Figure 3. Severe hemorrhage in COUP-TFII mutants. Shown are whole-mount views of embryos isolated from COUP-TFII heterozygote matings at E9.5 $(A-C)$ and E10.5 $(D-F)$. Hemorrhaging occurs in the mutant forebrain $(B$ and $C)$ and throughout the brain vesicles $(E)$ and heart $(F)$. Note the edematous cysts that are often found in mutants (arrows in C). Mutants at E10.5 are growth retarded and are being resorbed as compared with wild-type $(D)$. ( $\mathrm{Fb})$ Forebrain; $(\mathrm{Mb})$ midbrain; $(\mathrm{Hb})$ hindbrain; $(\mathrm{O})$ otocyst; (Lb) limbbud; (S) somite; (H) heart.

\section{Sinus venosus and atrial malformations in COUP-TFII mutants}

Next, we determined the likely cause of the hemorrhagic hearts, which is indicative of congestive heart failure. To facilitate the analyses of the mutant hearts, we used whole-mount platelet endothelial cell adhesion molecule-1 (PECAM) immunostaining to mark the endothelial cells. PECAM is expressed in all endothelial cells during cardiac and vascular development (Baldwin et al. 1994). In this manner, analysis of embryos between E8.5 and E10 revealed dramatic morphological defects in COUP-TFII mutants. Defects in heart development were easily apparent at E9 (Fig. 4). The extent of myocardial trabeculation in the wild-type ventricle was extensive as marked by PECAM immunostaining of the endothelium embedded in the myocardium (Fig. 4A). Most COUPTFII mutants had a relatively equivalent ventricular trabecular mass at E9, but some mutants had a reduced extent of trabeculation (Fig. 4B). The developing atrial chamber was relatively similar in size to the wild type at this stage. However, by E9.5, little expansion of the common atrial chamber demonstrates that atrial development has not progressed in mutant embryos (Fig. 4D). Serial cross sections through the hearts at E9.5 showed an extensive rightward expansion of the common atrial chamber in wild-type littermates (Fig. 4E), whereas COUP-TFII mutants only have a primitive tube (Fig. 4F) situated on the left. The right ventricle is thus misplaced dorsally and is directly in apposition to the foregut (Fig. $4 \mathrm{~F})$. The sinus venosus is comprised of enlarged bilateral chambers situated posterior and ventral to the common atrium in wild-type littermates (Fig. 4G). In COUP-TFII mutants, the primitive atrial tube is connected to an equally small and primitive sinus venosus (Fig. $4 \mathrm{H}$ ) that is situated over the midline. Because of the small size of the sinus venosus in mutants, the pericardial-peritoneal cavity is empty ( $\mathrm{p}$ in $4 \mathrm{H}$ ) whereas in wild-type littermates it is filled with the enlarged chambers of the sinus venosus (p in Fig. 4G). Because development and differentiation of the myocardium requires reciprocal inductive signaling between the endocardial and myocardial compartments, we analyzed for the normal differentiation of the myocardium using smooth muscle $\alpha$-actin (SMA) as a marker (Ruzicka and Schwartz 1988; Hungerford et al. 1996). Immunostaining for SMA revealed expression in all myocardial structures including the sinus venosus in the wild type (Fig. 4G) and in COUP-TFII

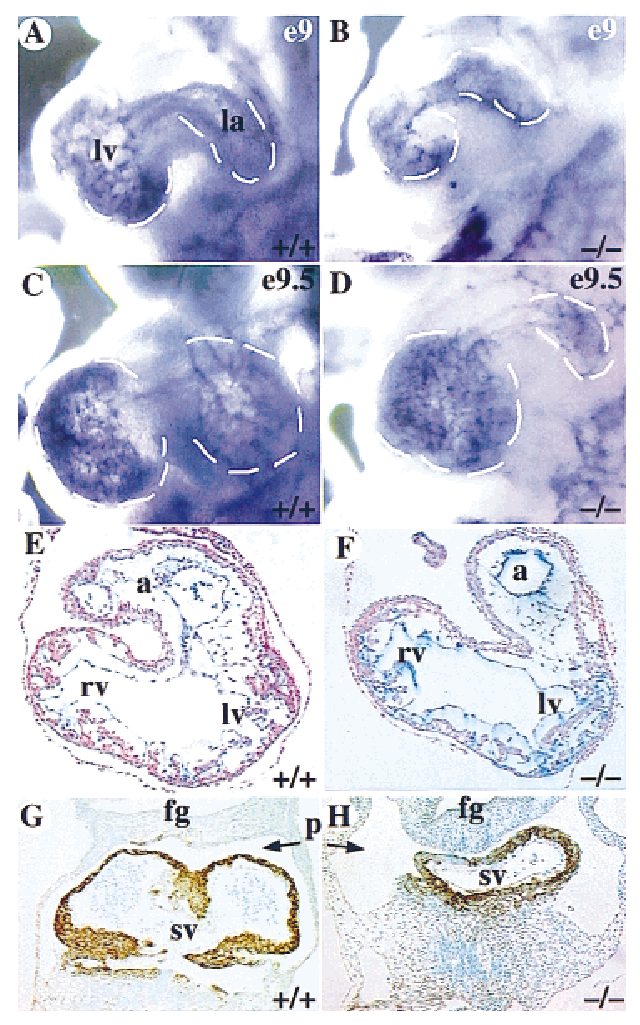

Figure 4. Defects in development of common atrium and sinus venosus. $(A-D)$ Whole-mount PECAM immunostaining of hearts showing the left ventricle (lv) and atrium (la), which are demarcated by dashed lines. There is reduced staining in mutant ventricles at E9 $(B)$, reflecting a reduced trabeculation in some mutant hearts. $(C-D)$ At E9.5, there is a clear enlargement of the atrium in the wild type $(C)$ whereas the mutant atrium is similar in size to an E9 embryo $(D)$. $(E-F)$ Sections through hearts showing an enlarged common atrial chamber (a) in the wild type $(E)$ but there is only a primitive atrial tube in the mutant $(F) .(G-H)$ Abundant SMA immunostaining in the myocardium of the bilaterally symmetrical sinus venosus $(\mathrm{Sv})$ that completely fills the pericardial-peritoneal cavity (p) in wild type $(G)$. In the mutant, SMA staining shows the sinus venosus is a narrow tube leaving most of the pericardial-peritoneal cavity vacant $(H)$. (fg) Foregut; (rv) right ventricle. 
mutant hearts (Fig. 4H). Thus, despite the defects in morphogenesis of the common atrium and sinus venosus, the myocardium is differentiating appropriately with respect to expression of SMA. However, the reduced size of both the common atrium and sinus venosus may lead to cardiac stress and would ultimately be detrimental to efficient circulation of blood in the rapidly developing embryo. These defects correlate directly with COUPTFII expression in the sinus venosus and atria at this and earlier developmental stages (see Fig. 1).

\section{Malformation of cardinal veins and disruption of systemic circulation in COUP-TFII mutants}

The developing systemic circulation at the E9-E10 stage is a simple system (Ulrich 1995). Oxygenation occurs in the branchial arches. The ventrally situated cardiac loop pumps the blood rostrally via the ventral aorta into the aortic arches that drain into the descending dorsal aorta. The dorsal aorta branches into the intersomitic arteries in the spine. Venous return is via the anterior and posterior cardinal veins that join into a common trunk, which opens into the sinus venosus and the atrium without flowing through the primitive liver bud. Wholemount PECAM staining revealed a substantial lumen in the dorsal aorta and anterior cardinal vein in wild-type embryos (Fig. 5A). Analysis of COUP-TFII mutants revealed a lack of any lumen in the anterior cardinal vein (Fig. 5, B, and arrow in D) or the posterior cardinal vein (Fig. 5, cf. arrows in $\mathrm{F}$ and $\mathrm{H}$ with same positions in $\mathrm{E}$ and G). Sometimes, a considerably reduced lumen in the anterior cardinal vein was present on one side of the animal (acv on right side of embryo in Fig. 5D) as compared with wild-type littermates (Fig. 5C). Sections through embryos at $\mathrm{E} 8.75$ revealed development of the primitive anterior cardinal veins with blood in the lumen (data not shown) but by E9-E9.5, both the anterior and posterior cardinal veins were collapsed or missing. Some cells were marked by PECAM, reflecting the presence of endothelial cells, but they did not encompass a lumen (data not shown). Thus, these results suggest either that the cardinal veins were formed but the lumen was not maintained or that the veins were malformed and readily collapsed. Nevertheless, collapse of the veins may be secondary to the decreased flow caused by the cardiac defects. The reduced venous return and the narrowed sinus venosus and atrial canal would clearly contribute to an altered systemic circulation and effective blood flow, which would ultimately lead to cardiac distress and failure.

\section{Abnormal angiogenesis in COUP-TFII mutants}

Formation of the embryonic vasculature involves two major steps, vasculogenesis and angiogenesis. The major vessels in COUP-TFII mutants, which arise through vasculogenesis, were formed and expressed the appropriate markers of endothelial lineage and differentiation, VEGF-R1, VEGF-R2 (data not shown), and PECAM (see
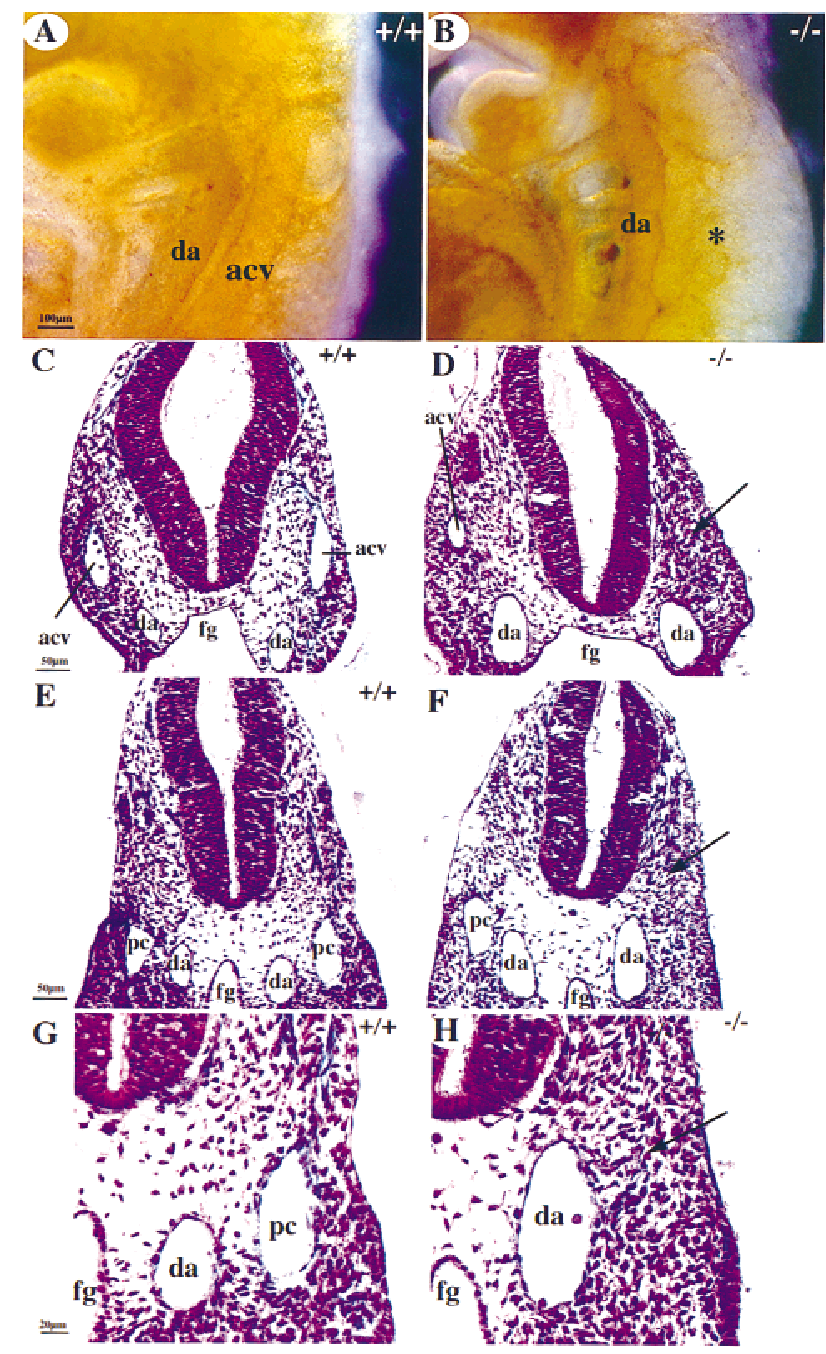

Figure 5. Malformation of cardinal veins $(A, B)$ Whole-mount PECAM immunostaining showing a lack of the anterior cardinal vein $(\mathrm{acv})$ in COUP-TFII mutants (asterisk in $B$ ) at E9.5. $(C-H)$ Cross sections at the level of the otic vesicle $(C, D)$ or at the midgut level $(E-H)$. There is a lack of acv (arrow in $D$ on left side of mutant embryo) and posterior cardinal vein (pc, arrow in $F$ and $H$ ) or a reduced luminal diameter in the acv in $D$ of mutants (right). $(G, H)$ High magnifications of $E$ and $F$. (da) Dorsal aorta; (fg) foregut.

Fig. 4A,B), indicating that vasculogenesis was essentially intact. The process of angiogenesis involves the sprouting of new vessels from pre-existing vessels to form a capillary network. Because angiogenesis and blood vessel remodeling are extensive in the early brain and spine, we used whole-mount PECAM immunostaining to analyze the extent, organization, and remodeling of the plexus.

The perineural plexus appears as a honeycomb structure of interconnected vessels of fairly uniform length and diameters throughout the early brain (Risau 1997). In the wild-type E9 brain, the perineural vascular plexus had already been extensively remodeled (Fig. 6A). There was remodeling of smaller vessels into larger vessels to form the branches of the anterior cardinal vein (arrow- 


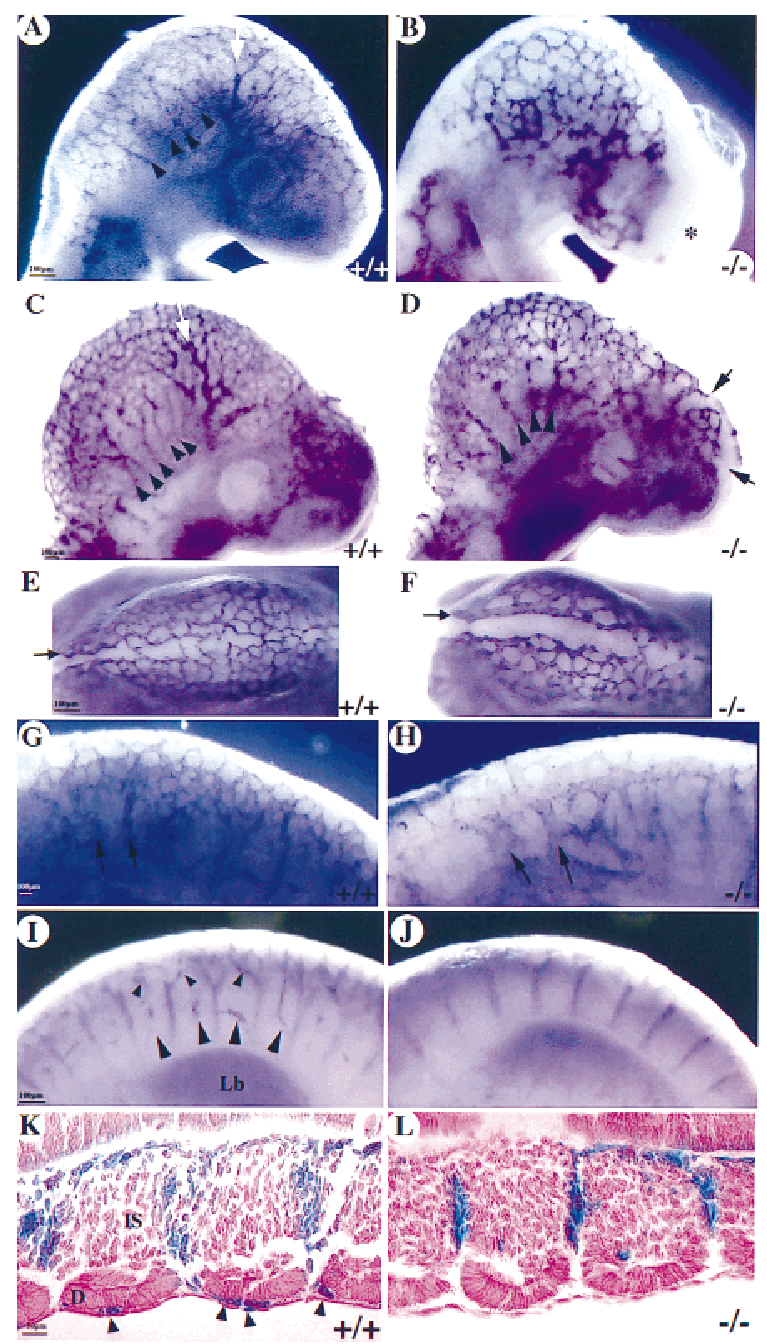

Figure 6. Angiogenesis defects. (A) A significantly remodeled vascular plexus in the wild type with formation of internal carotid artery (white arrow) and multiple branches of anterior cardinal vein (arrowheads) as revealed by whole-mount PECAM immunostaining. $(B)$ There is only a primitive plexus in the mutant brain at E9 with vessels devoid of significant remodeling. Note the lack of vessels, irregular growth (asterisk), and edematous bubble in the frontonasal region. $(C-L)$ At E9.5, there is continued vascular remodeling in the wild-type head (sagittal $C$; top $E$ views), in the caudal hindbrain $(G)$, and in the spine $(I, K)$. In the mutant, there is poor remodeling, and some vessels have varicosities in the head (arrowheads in $D$ ), are distended (arrows in $F, H$ ), or are poorly branched (arrows in $D$ and in the intersomitic region in $J$ and $L)$. $(K, L)$ Wild-type and COUP-TFII mutants heterozygous for Tie1-LacZ and stained for $\beta$-gal (frontal sections) showing the lack of any vessels over the somites in mutants $(L)$. (Lb) Limb bud; (D) dermamyotome; (IS) intersomitic vessels.

heads in Fig. 6A) and the internal carotid artery (white arrow in Fig. 6A). The perineural plexus in COUP-TFII mutants remained syncitial and appeared like vessels in the primitive honeycomb structure normally found at E8.75 (Fig. 6B) but with endothelial tubes of inappropriate lengths and diameters. There was delayed or no de- velopment of the internal carotid artery and few signs of remodeling resulting in a reduced complexity of the vascular plexus. This result suggests that remodeling of the vascular plexus was defective. By E9.5, remodeling was significantly progressed in the wild-type (Fig. 6C). The internal carotid artery was clearly apparent (white arrow in Fig. $6 \mathrm{C}$ ), and there were many distinct branches of the anterior cardinal vein (arrowheads in Fig. 6C). In contrast, there was still little remodeling of the plexus, resulting in vasodilation in some areas of the mutant brain (Fig. 6D, and cf. vessels at arrows in $\mathrm{E}$ and $\mathrm{G}$ with $\mathrm{F}$ and $\mathrm{H}$, respectively) and in the caudal hindbrain (Fig. 6, cf. arrows in $\mathrm{G}$ and $\mathrm{H}$ ). In addition, the limited number of anterior cardinal vein branches were partially collapsed, appearing as varicosities along their length (arrowheads in Fig. 6D). The internal carotid artery was still not formed at this stage (Fig. 6, cf. white arrow in A and C with same positions in $\mathrm{B}$ and $\mathrm{D}$ ). These data suggest that remodeling of the vascular plexus is impaired in COUPTFII mutants.

Inefficient angiogenesis in mutants was also readily apparent in the forebrain, where large regions of tissue were without any vascularization (Fig. 6, asterisk in B, arrows in D). The lack of vasculature in these regions reflects an inadequate capillary sprouting of new vessels or capillary regression in the naive forebrain tissue. This phenotype was also seen in the dorsal midbrain region, where there were many vessel sprouts crossing the midline in the wild type (Fig. 6E) but there were few vessel sprouts crossing the midline in mutants (Fig. 6F). At the level of the forelimb in the spine, there were also many vessels sprouting and branching over the somites in the wild-type (Fig. 6, arrowheads in I and K). There were essentially no vessel sprouts or branches over the somites in mutants (Fig. 6J,L). Taken together, our results indicate that angiogenesis is defective in COUPTFII mutants.

\section{Suppression of Ang1 expression in COUP-TFII mutants}

Some of the defects in vascular and heart development in mice lacking ANG1 or its receptor TIE2 (Sato et al. 1995; Suri et al. 1996; Patan 1998) correlate very well with phenotypes in COUP-TFII mutants. Similarities include vasodilation, failure to remodel the primitive plexus, and atrial and sinus venosus defects. The malformed cardinal veins are similar to those observed during ectopic overexpression of ANG2, which is thought to antagonize TIE2 signaling (Maisonpierre et al. 1997). Furthermore, ANG1 is highly expressed in mesenchymally derived cells (Davis et al. 1996; Suri et al. 1996; Patan 1998), where COUP-TFII is highly expressed (see Fig. 1). On the basis of the above observations, we hypothesized that COUP-TFII may function through the ANG1-TIE2 pathway. To address this possibility, we analyzed the expression of Ang1 in COUP-TFII mutants. We observed that Angl was highly expressed in the sinus venosus and myocardium leading into the common atrium in wildtype embryos at E9 (data not shown). At E9.5, it was 
found in the mesenchymal compartment in the frontonasal process, in the periocular and intersomitic regions, and throughout the ventricles, atria, and sinus venosus (Fig. 7A). The COUP-TFII expression pattern overlaps extensively with this Ang1 expression profile, especially in the atria, sinus venosus, brain, and intersomitic regions (cf. Figs. 7A and 1B). Interestingly, at all stages analyzed, Ang1 was significantly down-regulated in the mesenchyme of the brain, eye, somites, and heart in COUP-TFII mutants (Fig. 7B) as determined by wholemount in situ hybridization analysis. In contrast, there was appropriate expression of the homeobox gene Nkx2.5 in mutant hearts and VEGF-R2 in all mutant endothelia at this E9-E9.5 stage (data not shown). Finally, Ang1 expression was not completely lost in the ventricles, a site of minimal COUP-TFII expression, possibly explaining why trabeculation defects in COUPTFII mutants were less severe than in Ang1 mutants.

To determine the relative residual quantities of Ang1 transcripts in the heart, we used semiquantitative mimic RT-PCR analysis (Kramnik et al. 1993). The relative quantities of wild-type and mutant heart cDNAs were normalized to the L19 ribosomal protein RNA. This normalization was accomplished by amplification of an endogenous L19 fragment and twofold serial dilutions of a L19 mimic fragment (bottom panel in Fig. 7C, cf. lanes 3 and 4 with 8 and 9). The mimic fragment is a synthetic

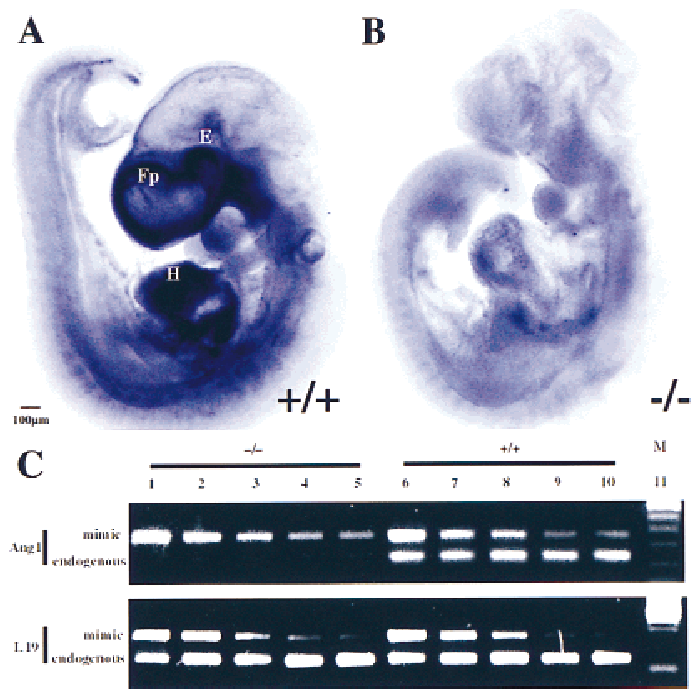

Figure 7. Down-regulation of Ang1 in COUP-TFII mutants. $(A-B)$ Whole-mount in situ hybridization of Ang1 in wild type $(A)$ and mutant $(B)$ at E9.5. There is abundant Ang1 expression throughout the heart $(\mathrm{H})$, frontonasal process $(\mathrm{Fp})$, and periocular mesenchyme (E) in the wild type $(A)$. In the COUP-TFII mutant, there is a dramatic reduction of Ang1 transcript in the frontonasal process and periocular mesenchyme and a significantly reduced expression in the ventricles and spine $(B) .(C)$ Semiquantitative mimic RT-PCR analysis of Ang1 transcripts in wild-type $(+/+)$ and mutant $(-/-)$ hearts as normalized to the level of L19 transcripts. Each lane represents amplified products of the endogenous gene and twofold serial dilution of the respective mimic plasmid. Note the 8- to 10 -fold decrease of endogenous Ang1 transcripts in the mutant. (M) Size markers. construct of nonsense DNA flanked by an identical primer complement pair as the endogenous L19 target. Thus, similar ratios of amplified products of endogenous L19 versus its mimic fragment from wild-type and mutant RNAs indicate that they have equivalent concentrations of L19 mRNA. By use of equivalent quantities of cDNA as determined by L19 amplification, endogenous Ang1 transcripts and serial twofold dilutions of an Ang1 mimic fragment were amplified from wild-type and COUP-TFII mutant hearts. A significant and consistent 8- to 10-fold decrease in endogenous Ang1 transcripts was observed in COUP-TFII mutant hearts (top panel in Fig. 7C, cf. lanes 4 and 5 with 9 and 10), which confirmed the whole-mount in situ analysis. Thus, down-regulation of ANG1 expression may substantially interfere with signaling through the ANG1-TIE2 pathway, leading to the heart and vascular defects observed in COUPTFII mutants.

\section{Discussion}

The genetic and molecular mechanisms that control the processes of vascular and heart development are currently being elucidated. We have described a new effector, COUP-TFII, which plays a regulatory role in angiogenesis and heart development. COUP-TFI and COUPTFII are orphan members of the nuclear receptor superfamily of ligand-activated transcription factors. Although COUP-TFI and COUP-TFII have been shown to transcriptionally regulate some genes required for embryogenesis, such as BMP4 (Feng et al. 1995) and Oct3/4 (Schoorlemmer et al. 1995), and are well characterized biochemically, their precise physiological roles are largely undefined. The patterns of COUP-TFI and COUP-TFII expression overlap in development, but each factor possesses its own distinct profile. In addition, both genes are induced by retinoids (Jonk et al. 1994b; Fjose et al. 1995; Qiu et al. 1995) and COUP-TFII is regulated by Sonic hedgehog signaling (Krishnan et al. 1997a,b). Thus, the different expression profiles and regulation support the notion that each gene possesses a unique specific physiological role. Indeed, we showed previously that COUP-TFI mutants display defects in neurogenesis, axon guidance, and arborization (Qiu et al. 1997), and, now we describe mice lacking COUP-TFII with striking defects in angiogenesis, vascular remodeling, and fetal heart development. The defects in COUP-TFII mutants are consistent with its temporal and spatial expression pattern in the mesenchyme during embryogenesis and suggest that COUP-TFII may regulate aspects of mesenchymal-endothelial signaling.

\section{COUP-TFII is required for heart development}

Vertebrate heart formation involves the commitment of mesodermal precursor cells to the cardiac lineage and the subsequent formation of a primitive heart tube. The linear heart tube proliferates and undergoes looping, formation of the atrial and ventricular cavities, and septa- 
tion to form the mature four-chambered heart /Olson and Srivastava 1996; Fishman and Chien 1997; Fishman and Olson 1997). Little is known about the underlying genetic pathways that control cardiac atrial and sinus venosus development. Now, we show that the COUPTFII gene is required for proper atrial and sinus venosus development. Indeed, COUP-TFII mutant atria fail to expand to the extent found in wild-type littermates, and the connection to the sinus venosus proper remains primitive in nature. In addition, both anterior and posterior cardinal veins are either poorly formed or had collapsed, imposing a detrimental stress on the primitive heart and on the systemic circulation of the fast-growing embryo. Defects in these developing structures probably reflect the loss of COUP-TFII function in the precursor mesodermal cells, which may perturb signaling between the myocardium and endocardium. It is known that myocardial differentiation and development are intricately dependent on endocardium differentiation and development. Thus, the inappropriate signals may affect morphogenesis of the heart structures. The resulting narrowing of the blood flow in the sinus venosus and atria may lead to cardiac distress and contribute to the hemorrhage and lethality of the COUP-TFII mutants, the majority of which succumb at this stage.

To date, only mutations in the Ang1 or Tie2 genes have yielded atrial chamber and vascular development abnormalities as described in COUP-TFII mutants. Because both genes are expressed in the myocardium of the atria and sinus venosus, loss of COUP-TFII function in these cells is consistent with the down-regulation of Ang1. However, the defects in atrial and sinus venosus chamber development described here are more severe than those described for Ang1 (Suri et al. 1996). ANG2 antagonism of TIE2 signaling cannot account for the heart defects in COUP-TFII mutants because it is not normally found in the heart at this stage (Maisonpierre et al. 1997). Thus, the reduced signaling of this pathway in COUP-TFII mutant hearts is due in part to the loss of ANG1 expression. This conclusion suggests that other gene(s) important for atria and sinus venosus development must also be regulated by COUP-TFII. In addition, it would be interesting to determine whether any of the mutants defective in heart development identified in the large zebrafish screens result from mutation of any of the three COUP-TF homologs (Chen et al. 1996; Stainier et al. 1996).

\section{A role for COUP-TFII in vascular remodeling}

Formation of the primitive embryonic vasculature has been separated into two major processes, vasculogenesis and angiogenesis. The de novo differentiation of angioblasts and assembly of a primitive vascular plexus, vasculogenesis, are largely normal in COUP-TFII mutants. This normal state is indicated by the appropriate expression of the differentiation marker genes (VEGF-R1, VEGF-R2, and PECAM). Maturation and disassembly of the primitive plexus to form large and small vessels and the subsequent sprouting and branching to form a com- plex microcapillary network, angiogenesis, are largely impaired in COUP-TFII mutants. The inefficient remodeling of the primary vascular plexus, the reduced complexity of the resultant vascular network, and atrial defects are reminiscent of those described for mice lacking ANG1 or TIE2 or for mice overexpressing ANG2.

Yet, the defects in COUP-TFII mutants are not identical to the specific mutants of the ANG1-TIE2 pathway. Mutation of Ang1 results in a significant downregulation of its receptor TIE2 (Suri et al. 1996); however, we did not detect changes in Tie2 transcripts by wholemount or section in situ analyses in the absence of COUP-TFII (data not shown). This observation suggests that the basal ANG1 expression in COUP-TFII mutants is sufficient to maintain TIE2 expression but is insufficient to maintain effective vascular development. Although ANG2 overexpression results in similar phenotypes (Maisonpierre et al. 1997) to those observed in COUP-TFII mutants, its expression was unchanged in COUP-TFII mutants as determined by in situ analysis (data not shown). Also, a complete suppression of vascular remodeling in the yolk sacs has only been observed in about $1 \%$ of COUP-TFII mutants (data not shown). Because the defects we describe here are from independent lines and in different genetic backgrounds, the different phenotypes may be due to redundancy with COUP-TFI function or to the complexity in regulation of development of the vascular and heart systems. Taken together, similar to the role COUP-TFII plays in heart development, COUP-TFII must perturb signaling pathways in addition to the ANG1-TIE2 pathway to regulate vascular remodeling processes.

Because COUP-TFII mutants had extensive defects in angiogenesis and vascular remodeling, we analyzed many other genes implicated in regulating different aspects of these processes. The poor blood circulation, hemorrhage, and edema in the early COUP-TFII mutant brain may lead to hypoxia. VEGF is highly responsive to hypoxia (Stein et al. 1995) and, indeed, VEGF was upregulated (10-fold) in E9.5 COUP-TFII mutants, especially in the telencephalic region (data not shown). Overexpression of VEGF is characterized by endothelial leakiness and hyperpermeability (Drake and Little 1995; Okamoto et al. 1997; Detmar et al. 1998; Zeng et al. 1998), which would account for the edema in COUPTFII mutants. In addition, expression of other molecules (Tie1, PDGF-B, PDGF $\beta R$, TGF $\beta 1$, and HB-EGF) implicated in the processes of angiogenesis was unaltered in the E9.5 COUP-TFII mutant (data not shown). This result suggests that COUP-TFII may regulate a novel signal transduction pathway required for heart and vascular development. Thus, there must be other molecules to be discovered that regulate specific aspects of vessel formation or atrial and sinus venosus development.

The inability of COUP-TFII mutants to form the internal carotid artery and to remodel the primitive plexus into a complex microcapillary bed is similar to the recent findings observed on ablation of the Eph ligand, ephrin-B2 (Wang et al. 1998). However, preliminary in situ analysis of ephrin-B2 and its receptor Eph B4 revealed no 
significant differences in wild-type and COUP-TFII mutants at E9 (data not shown). Nevertheless, our results are consistent with the hypothesis that there are reciprocal interactions between developing arteries and veins that are vital to the vessel remodeling process. In addition, further perturbation of TIE2 signaling may occur as a result of the apparently normal expression of the receptor antagonist ANG2 in mutant tissues (Maisonpierre et al. 1997). Cumulatively, multiple mechanisms may affect the process of blood vessel assembly, resulting in decreased sprouting and branching and leading to a syncitial appearance of the plexus, poor integrity of the vessels, and inadequate remodeling in COUP-TFII mutants.

Many genes have been implicated in the process of blood vessel formation. A working model (Folkman and D'Amore 1996) suggests that proangiogenic factors (such as Ang1) secreted by periendothelial cell precursors, bind and activate a trans-membrane RTK (Tie2) on endothelial cells. Signaling from TIE2 induces endothelial cell proliferation and secretion of chemoattractants and mitogens such as PDGF-BB. In the milieu of small vessels, PDGF-BB stimulates its receptor on the mesenchymal cells and induces their proliferation and migration. Once a periendothelial cell precursor contacts the endothelial cell, TGF $\beta$ is proteolytically activated, thereby inducing matrix deposition, periendothelial cell differentiation, and growth suppression (Folkman and D'Amore 1996).

The aberrant formation of the vasculature in COUPTFII mutants suggests COUP-TFII either regulates mesenchymal cell recruitment and differentiation directly or regulates endothelial cells through paracrine signals. In either event, it is hypothesized that COUP-TFII is important for mesenchymal-endothelial interactions in embryonic vasculature formation. Our data suggest that COUP-TFII is one transcription factor that regulates high-level expression of the proangiogenic factor ANG1 in mesenchymal cells. Genetic ablation of COUP-TFII function results in decreased Ang1 expression. The resultant level of ANG1 is insufficient to maintain effective signaling through its receptor TIE2 on the endothelial cell. In addition, loss of a paracrine signal necessary to stimulate TIE2 signaling or persistent antagonism of TIE2 signaling would alter the ability of endothelial cells to respond effectively to proangiogenic signals. This situation would cause edema and unstable vessels that would hemorrhage in the embryo. Prolonged disruption of the vascular system results in hypoxia and elevated VEGF levels that induce further vascular permeability and edema. This compromised state is lethal to the rapidly growing embryo. Whether COUP-TFII acts autonomously to modulate the proliferation and differentiation of periendothelial cells directly in addition to acting nonautonomously to modulate endothelial cell differentiation and maturation through angiogenic paracrine signal(s) remains to be elucidated. Nevertheless, the defects observed in the COUP-TFII mutants are consistent with the hypothesis that COUP-TFII may play an important role in mesenchymal-endothelial interactions.

In conclusion, our results indicate that COUP-TFII function is required for mesenchymal-endothelial interactions during heart development, angiogenesis, and vascular remodeling in part through the ANG1-TIE2 pathway. Identification of other, possibly novel, pathways affected in COUP-TFII mutants is eagerly anticipated. In addition, ligand agonist(s) and antagonists for COUPTFII, which are characteristically small steroid-like, lipophilic molecules for this nuclear receptor family, may provide very important therapeutic tools in the treatment of diseases whose etiology is derived through improper angiogenesis. The ability to activate or repress COUP-TFII function will be a new tool to modulate the processes of angiogenesis in tumor growth and progression (Lee and Feldman 1998).

\section{Materials and methods}

Targeting vector construction

A mouse 129Sv genomic library (a gift from Dr. Phil Soriano, Fred Hutchinson Cancer Research Center) was screened with a mouse cDNA fragment containing the complete COUP-TFII open reading frame. Three positive clones encompassed the entire COUP-TFII gene, which contains three exons (Qiu et al. 1995). To create the $5^{\prime}$ homologous arm, a 2.3-kb (EcoRI-StuI) fragment containing the $5^{\prime}$-untranslated region was cloned into the EcoRI-HindIII (HindIII filled-in) site of pBluescript. The $P G K n e o b p A$ gene $\left(\right.$ neo $^{\mathrm{r}}$; Soriano et al. 1991) contained in a SalI$X$ hoI fragment was then inserted in the same transcriptional direction as the COUP-TFII gene in the Xhol site of this vector for positive selection. The $3^{\prime}$ homologous arm, which consisted of a $3-\mathrm{kb}$ fragment containing a major part of intron 2, was constructed from a 2.7-kb EcoRI-HindIII (HindIII filled-in) and a 0.3-kb EcoRI-SalI fragment. First, the 2.7-kb fragment was placed into the EcoRI-SmaI site of pBluescriptKS+. Then, the $0.3-\mathrm{kb}$ fragment was obtained from a $1.0-\mathrm{kb}$ StuI-EcoRI fragment that was cloned into the HindIII (blunt)/EcoRI site of this pBluescriptKS+ construct with subsequent removal of a $0.7-\mathrm{kb}$ SalI fragment. Addition of XhoI linkers destroyed the BamHI site of this vector. The vector was digested with SalI-XhoI and this $3^{\prime}$ construct subcloned into the Xhol site of the $5^{\prime} \mathrm{arm} / \mathrm{neo}^{\mathrm{r}}$ construct. The resulting construct was digested with XhoI-NotI (blunt ended) and cloned into the XhoI-BamHI (blunt ended) site of pSP72HSVTK, which contained the herpes simplex virus thymidine kinase gene for negative selection (Mansour et al. 1988). The targeting construct was linearized with XhoI before electroporation.

Electroporation, screening of G418-resistant ES colonies, and genotyping

Culturing, manipulation, and screening of ES cells were essentially as described previously (Qiu et al. 1997). For Southern analyses, the $5^{\prime}$ probe was a 0.8 -kb EcoRI fragment, and $X b a \mathrm{I}$ was used as the diagnostic enzyme as the neo ${ }^{\mathrm{r}}$ cassette introduced an extra $\mathrm{XbaI}$ site, yielding $10-\mathrm{kb}$ wild-type and 8 -kb mutant fragments. To check the 3' arm, a 1-kb EcoRV-NsiI 3' fragment downstream of the $3^{\prime}$ homologous sequence was used on SpeI digests. The expected $12-\mathrm{kb}$ wild-type and $6-\mathrm{kb}$ mutant bands were appropriately detected. In addition, genotyping of embryos at various gestational stages (E8-E10.5) was performed by isolation of yolk sac genomic DNA. After an overnight digestion in proteinase $\mathrm{K}$ buffer $[0.9 \mathrm{mg} / \mathrm{ml}$ proteinase $\mathrm{K}, 10 \mathrm{~mm}$ Tris (pH 7.8), $10 \mathrm{~mm} \mathrm{NaCl}, 1 \mathrm{~mm}$ EDTA, 0.5\% Triton X-100], 
the enzyme was heat inactivated at $90^{\circ} \mathrm{C}$ for $10 \mathrm{~min}$, and the DNA was used directly for PCR. For the wild-type allele, the $5^{\prime}$ primer (5'-AGCTTCTCCACTTGCTCTTGG-3') was located in exon 2 and was deleted in the mutant allele. The $3^{\prime}$ primer (5'-CAGCTGTACAGAGAGGCAGG-3') was located at the end of exon 2 and was $3^{\prime}$ to the SalI site. These primers generated a 466-bp wild-type fragment. To detect the mutant allele, the $3^{\prime}$ primer (5'-GATATGGCAATGGTAGTCAGC-3'), located at the $3^{\prime}$ end of the PGKneobpA gene, and 5' primer (5'-GTGCTGTCCATCTGCACGAG-3') located near the amino terminus of the gene were used to generate a 376-bp fragment. PCR was performed according to the manufacturer's instructions (Promega) with $1.2 \mathrm{mM} \mathrm{MgCl}_{2}$ at an annealing temperature of $55^{\circ} \mathrm{C}$.

\section{Generation of chimeric mice and breeding}

Clones with appropriately recombined COUP-TFII loci were thawed, expanded, and injected into E3.5 C57BL/6 blastocysts. Injected embryos were transferred into the uteri of pseudopregnant $\mathrm{F}_{1}(\mathrm{CBA} \times \mathrm{C} 57 \mathrm{BL} / 6)$ foster mothers. Male chimeras with $80 \%-100 \%$ agouti coat color were backcrossed with C57BL/6 females, and germ-line transmission was determined by the presence of agouti offspring. COUP-TFII heterozygotes were determined by Southern or PCR analysis and intercrossed to generate homozygotes.

\section{Whole-mount and section in situ hybridization}

Whole-mount or section in situ hybridization was performed with a 1-kb (NsiI-EcoRV) COUP-TFII 3' UTR cDNA fragment or an Ang1 cDNA fragment (from G.D. Yancopoulos, Regeneron, Tarrytown, NY) as templates for transcription of digoxigenin-labeled (whole mount) or $\left[{ }^{35} \mathrm{~S}\right] \mathrm{UTP}$-labeled (section) antisense riboprobes. The protocols used were essentially as published previously (Qiu et al. 1994, 1997).

\section{Immunohistochemistry}

Whole-mount immunohistochemistry to mark endothelial cells was accomplished with a monoclonal antibody (MEC 13.1, PharMingen) recognizing PECAM/CD31 and was performed as described previously with modifications (Qiu et al. 1997). Briefly, embryos were collected in PBS and fixed in methanol/ DMSO $(4 / 1)$ overnight at $4^{\circ} \mathrm{C}$. Then, the embryos were bleached in methanol/DMSO/30\% $\mathrm{H}_{2} \mathrm{O}_{2}(4 / 1 / 1)$ for $4-5 \mathrm{hr}$ at room temperature, rehydrated for $30 \mathrm{~min}$ each through $50 \%$ and $15 \%$ methanol, and, finally, washed in PBS. Embryos were incubated twice in PBT.3 [1\% blocking reagent (Boehringer Mannheim) and $0.3 \%$ Triton $\mathrm{X}-100$ in PBS] for $1 \mathrm{hr}$ at room temperature. Then, they were incubated with the diluted primary antibody $(1 / 50)$ in PBT.3 with $2 \%$ blocking reagent (BR; Boehringer Mannheim) at $4^{\circ} \mathrm{C}$ overnight. Embryos were washed twice briefly in PBT.3 at room temperature and then in PBT.5 (PBT with 0.5\% Triton X-100) at room temperature for $1 \mathrm{hr}$ each. These washes were followed by an overnight incubation at $4^{\circ} \mathrm{C}$ with alkaline phosphatase-conjugated goat anti-rat IgG diluted 1/200 in PBS.3 with $2 \% \mathrm{BR}$. The washes were similar to post-primary antibody washes with an additional 20-min wash in CT.3 buffer [100 mM Tris (pH 9.5), $150 \mathrm{~mm} \mathrm{NaCl}, 25 \mathrm{~mm} \mathrm{MgCl}_{2}, 0.3 \%$ Triton X-100]. For color development, embryos were incubated in $3.5 \mu \mathrm{l} / \mathrm{ml}$ BCIP, $4.5 \mu \mathrm{l} / \mathrm{ml} \mathrm{NBT}$, and $10 \%$ polyvinyl alcohol in CT buffer (no detergent) to the desired intensity at room temperature. Then embryos were rinsed in PBT.3 to stop the reaction, dehydrated through a methanol series $(30 \%, 50 \%, 80 \%$, and $100 \%$ for $10 \mathrm{~min}$ each), and cleared in glycerol/PBS (50/50) for pho- tography. Embryos were photographed by use of a Zeiss Stemi 2000 dissecting microscope.

\section{$R T-P C R$}

Total RNA was isolated from wild-type or COUP-TFII mutant hearts by use of Trizol reagent (GIBCO-BRL). Samples (5 $\mu \mathrm{g}$ ) were reverse transcribed with oligo(dT) and Superscript II (MMLV-RT; GIBCO-BRL). RT-PCR detection of wild-type or mutant transcripts from E9.5 day embryos used the same primer sets as those used for PCR genotyping. To ensure that amplified fragments represented products of a reverse transcription reaction, a control reaction lacking the reverse transcriptase was used. For semiquantitative mimic RT-PCR, the relative amounts of mRNA for Angl was determined by use of L19 ribosomal protein mRNA to normalize the amounts of total cDNAs derived from wild-type and mutant samples. Usually, $5 \%$ of the reverse transcription reaction was co-amplified with specific amounts of mimic DNA templates, which were diluted serially (four consecutive twofold dilutions). Specific mimic DNA templates were constructed with primer sets for Ang1 (5'-CAGGCTCCACGCTGAACGGTTA-3' and 5'-CCTCCTGCAAAGCTTGCTCTTCTCT-3') or L-19 (5' -CTGAAGGTCAAAGGGAATGTG-3' and 5'-GGACAGAGTCTTGATGATCTC-3') separated by stuffer DNA (pBluescript). Thus, each set of primers would specifically amplify the mimic DNA template as efficiently as the endogenous target cDNA. PCR amplification was done in $25 \mu \mathrm{l}$ of PCR buffer [10 mM Tris (pH 8.2), $50 \mathrm{~mm}$ $\mathrm{KCl}, 1.5 \mathrm{mM} \mathrm{MgCl}_{2}$, and $200 \mu \mathrm{M}$ of each dNTP containing 2.5 units of Taq polymerase (Boehringer Mannheim)]. Amplification occurred by repeated cycles (usually 30 cycles) at $94^{\circ} \mathrm{C}$ for $1 \mathrm{~min}, 57^{\circ} \mathrm{C}$ for $1 \mathrm{~min}$, and $72^{\circ} \mathrm{C}$ for $1 \mathrm{~min}$, with a final extension at $72^{\circ} \mathrm{C}$ for $5 \mathrm{~min}$. Twenty percent of the reaction products was subjected to electrophoresis for analysis.

\section{Acknowledgments}

We would like to thank Carena L. Chai, as well as John Ash, Austin Cooney, Karen K. Hirschi, Mark W. Majesky, and John J. Schwarz, for critique of the manuscript. We thank Dr. F.J. DeMayo for generating the chimeras and Christopher T. Lee, Naomi Lee, and Mei-jin Chu for technical assistance. We thank Dr. G.D. Yancopoulos and Regeneron Pharmaceuticals for use of Ang1, Ang2, and Tie1. For probes we thank D.J. Anderson (ephrin-B2 and EphB4), W. Risau (VEGF), G. Breier (Flk-1), D. Dumont (Tek), J. Cook (Flt20), P. Soriano (PDGF $\beta R$ ), G. Merlino (TGFß1), D.F. Bowen-Pope (PDGF-B), C. Funk (HB-EGF), and R.J. Schwartz (Nkx2.5). Y.Q. was supported by a U.S. Public Health Service grant and F.A.P. by a fellowship from the Medical Research Council of Canada. This work was supported by grants from the National Institutes of Health to S.Y.T. and M.J.T. and from the American Heart Association (Texas affiliate) to F.A.P.

The publication costs of this article were defrayed in part by payment of page charges. This article must therefore be hereby marked 'advertisement' in accordance with 18 USC section 1734 solely to indicate this fact.

\section{References}

Baldwin, H.S., H.M. Shen, H.C. Yan, H.M. DeLisser, A. Chung, C. Mickanin, T. Trask, N.E. Kirschbaum, P.J. Newman, S.M. Albelda et al. 1994. Platelet endothelial cell adhesion molecule-1 (PECAM-1/CD31): Alternatively spliced, function- 
ally distinct isoforms expressed during mammalian cardiovascular development. Development 120: 2539-2553.

Chen, J.N., P. Haffter, J. Odenthal, E. Vogelsang, M. Brand, F.J. van Eeden, M. Furutani-Seiki, M. Granato, M. Hammerschmidt, C.P. Heisenberg, Y.J. Jiang, D.A. Kane, R.N. Kelsh, M.C. Mullins, and C. Nüsslein-Volhard. 1996. Mutations affecting the cardiovascular system and other internal organs in zebrafish. Development 123: 293-302.

Davis, S., T.H. Aldrich, P.F. Jones, A. Acheson, D.L. Compton, V. Jain, T.E. Ryan, J. Bruno, C. Radziejewski, P.C. Maisonpierre, and G.D. Yancopoulos. 1996. Isolation of angiopoietin-1, a ligand for the TIE2 receptor, by secretion-trap expression cloning. Cell 87: 1161-1169.

Detmar, M., L.F. Brown, M.P. Schon, B.M. Elicker, P. Velasco, L. Richard, D. Fukumura, W. Monsky, K.P. Claffey, and R.K. Jain. 1998. Increased microvascular density and enhanced leukocyte rolling and adhesion in the skin of VEGF transgenic mice. J. Invest. Dermatol. 111: 1-6.

Drake, C.J. and C.D. Little. 1995. Exogenous vascular endothelial growth factor induces malformed and hyperfused vessels during embryonic neovascularization. Proc. Nat1. Acad. Sci. 92: 7657-7661.

Dumont, D.J., G. Gradwohl, G.H. Fong, M.C. Puri, M. Gertsenstein, A. Auerbach, and M.L. Breitman. 1994. Dominantnegative and targeted null mutations in the endothelial receptor tyrosine kinase, tek, reveal a critical role in vasculogenesis of the embryo. Genes \& Dev. 8: 1897-1909.

Feng, J.Q., D. Chen, A.J. Cooney, M.J. Tsai, M.A. Harris, S.Y. Tsai, M. Feng, G.R. Mundy, and S.E. Harris. 1995. The mouse bone morphogenetic protein-4 gene. Analysis of promoter utilization in fetal rat calvarial osteoblasts and regulation by COUP- TFI orphan receptor. I. Biol. Chem. 270: 28364-28373.

Fishman, M.C. and K.R. Chien. 1997. Fashioning the vertebrate heart: Earliest embryonic decisions. Development 124: 2099-2117.

Fishman, M.C. and E.N. Olson. 1997. Parsing the heart: Genetic modules for organ assembly. Cell 91: 153-156.

Fjose, A., S. Nornes, U. Weber, and M. Mlodzik. 1993. Functional conservation of vertebrate seven-up related genes in neurogenesis and eye development. EMBO J. 12: 1403-1414.

Fjose, A., U. Weber, and M. Mlodzik. 1995. A novel vertebrate svp-related nuclear receptor is expressed as a step gradient in developing rhombomeres and is affected by retinoic acid. Mech. Dev. 52: 233-246.

Folkman, J. and P.A. D'Amore. 1996. Blood vessel formation: What is its molecular basis? Cell 87: 1153-1155.

Hanahan, D. 1997. Signaling vascular morphogenesis and maintenance. Science 277: 48-50.

Hungerford, J.E., G.K. Owens, W.S. Argraves, and C.D. Little. 1996. Development of the aortic vessel wall as defined by vascular smooth muscle and extracellular matrix markers. Dev. Biol. 178: 375-392.

Jonk, L.J., M.E. de Jonge, C.E. Pals, S. Wissink, J.M. Vervaart, J. Schoorlemmer, and W. Kruijer. 1994a. Cloning and expression during development of three murine members of the COUP family of nuclear orphan receptors. Mech. Dev. 47: 81-97.

Jonk, L.J., M.E. de Jonge, J.M. Vervaart, S. Wissink, and W. Kruijer. 1994b. Isolation and developmental expression of retinoic-acid-induced genes. Dev. Biol. 161: 604-614.

Kramnik, I., E. Skamene, and D. Radzioch. 1993. Assessment of lymphokine profiles in activated lymphocytes by semiquantitative PCR. J. Immunol. Methods 162: 143-153.

Krishnan, V., G. Elberg, M.J. Tsai, and S.Y. Tsai. 1997a. Identification of a novel sonic hedgehog response element in the chicken ovalbumin upstream promoter-transcription factor II promoter. Mol. Endocrinol. 11: 1458-1466.

Krishnan, V., F.A. Pereira, Y. Qiu, C.H. Chen, P.A. Beachy, S.Y. Tsai, and M.J. Tsai. 1997b. Mediation of Sonic hedgehoginduced expression of COUP-TFII by a protein phosphatase. Science 278: 1947-1950.

Lee, J.S. and A.M. Feldman. 1998. Gene therapy for therapeutic myocardial angiogenesis: A promising synthesis of two emerging technologies. Nat. Med. 4: 739-742.

Lee, K.F., H. Simon, H. Chen, B. Bates, M.C. Hung, and C. Hauser. 1995. Requirement for neuregulin receptor erbB2 in neural and cardiac development. Nature 378: 394-398.

Lutz, B., S. Kuratani, A.J. Cooney, S. Wawersik, S.Y. Tsai, G. Eichele, and M.J. Tsai. 1994. Developmental regulation of the orphan receptor COUP-TF II gene in spinal motor neurons. Development 120: 25-36.

Maisonpierre, P.C., C. Suri, P.F. Jones, S. Bartunkova, S.J. Wiegand, C. Radziejewski, D. Compton, J. McClain, T.H. Aldrich, N. Papadopoulos, T.J. Daly, S. Davis, T.N. Sato, and G.D. Yancopoulos. 1997. Angiopoietin-2, a natural antagonist for Tie2 that disrupts in vivo angiogenesis. Science 277: 55-60.

Meyer, D. and C. Birchmeier. 1995. Multiple essential functions of neuregulin in development. Nature 378: 386-390.

Mlodzik, M., Y. Hiromi, U. Weber, C.S. Goodman, and G.M. Rubin. 1990. The Drosophila seven-up gene, a member of the steroid receptor gene superfamily, controls photoreceptor cell fates. Cell 60: 211-224.

Okamoto, N., T. Tobe, S.F. Hackett, H. Ozaki, M.A. Vinores, W. LaRochelle, D.J. Zack, and P.A. Campochiaro. 1997. Transgenic mice with increased expression of vascular endothelial growth factor in the retina: A new model of intraretinal and subretinal neovascularization. Am. J. Pathol. 151: 281-291.

Olson, E.N. and D. Srivastava. 1996. Molecular pathways controlling heart development. Science 272: 671-676.

Patan, S. 1998. TIE1 and TIE2 receptor tyrosine kinases inversely regulate embryonic angiogenesis by the mechanism of intussusceptive microvascular growth. Microvasc. Res. 56: $1-21$.

Pereira, F.A., Y. Qiu, M.J. Tsai, and S.Y. Tsai. 1995. Chicken ovalbumin upstream promoter transcription factor (COUP$\mathrm{TF})$ : Expression during mouse embryogenesis. J. Steroid Biochem. Mol. Biol. 53: 503-508.

Qiu, Y., A.J. Cooney, S. Kuratani, F.J. DeMayo, S.Y. Tsai, and M.J. Tsai. 1994. Spatiotemporal expression patterns of chicken ovalbumin upstream promoter-transcription factors in the developing mouse central nervous system: Evidence for a role in segmental patterning of the diencephalon. Proc. Nat1. Acad. Sci. 91: 4451-4455.

Qiu, Y., V. Krishnan, Z. Zeng, D.J. Gilbert, N.G. Copeland, L. Gibson, T. Yang-Feng, N.A. Jenkins, M.J. Tsai, and S.Y. Tsai. 1995. Isolation, characterization, and chromosomal localization of mouse and human COUP-TF I and II genes. Genomics 29: 240-246.

Qiu, Y., F.A. Pereira, F.J. DeMayo, J.P. Lydon, S.Y. Tsai, and M.J. Tsai. 1997. Null mutation of mCOUP-TFI results in defects in morphogenesis of the glossopharyngeal ganglion, axonal projection, and arborization. Genes \& Dev. 11: 1925-1937.

Risau, W. 1997. Mechanisms of angiogenesis. Nature 386: 671674.

Risau, W. and I. Flamme. 1995. Vasculogenesis. Annu. Rev. Cell Dev. Biol. 11: 73-91.

Ruzicka, D.L. and R.J. Schwartz. 1988. Sequential activation of alpha-actin genes during avian cardiogenesis: Vascular smooth muscle alpha-actin gene transcripts mark the onset of cardiomyocyte differentiation. I. Cell Biol. 
107: 2575-2586.

Sato, T.N., Y. Tozawa, U. Deutsch, K. Wolburg-Buchholz, Y. Fujiwara, M. Gendron-Maguire, T. Gridley, H. Wolburg, W. Risau, and Y. Qin. 1995. Distinct roles of the receptor tyrosine kinases Tie-1 and Tie-2 in blood vessel formation. $\mathrm{Na}$ ture 376: 70-74.

Schoorlemmer, J., L. Jonk, S. Sanbing, A. van Puijenbroek, A. Feijen, and W. Kruijer. 1995. Regulation of Oct-4 gene expression during differentiation of EC cells. Mol. Biol. Rep. 21: 129-140.

Stainier, D.Y., B. Fouquet, J.N. Chen, K.S. Warren, B.M. Weinstein, S.E. Meiler, M.A. Mohideen, S.C. Neuhauss, L. Solnica-Krezel, A.F. Schier, F. Zwartkruis, D.L. Stemple, J. Malicki, W. Driever, and M.C. Fishman. 1996. Mutations affecting the formation and function of the cardiovascular system in the zebrafish embryo. Development 123: 285-292.

Stein, I., M. Neeman, D. Shweiki, A. Itin, and E. Keshet. 1995. Stabilization of vascular endothelial growth factor mRNA by hypoxia and hypoglycemia and coregulation with other ischemia-induced genes. Mol. Cell. Biol. 15: 5363-5368.

Suri, C., P.F. Jones, S. Patan, S. Bartunkova, P.C. Maisonpierre, S. Davis, T.N. Sato, and G.D. Yancopoulos. 1996. Requisite role of angiopoietin-1, a ligand for the TIE2 receptor, during embryonic angiogenesis. Cell 87: 1171-1180.

Tsai, M.J. and B.W. O'Malley. 1994. Molecular mechanisms of action of steroid/thyroid receptor superfamily members. Annu. Rev. Biochem. 63: 451-486.

Tsai, S.Y. and M.J. Tsai. 1997. Chick ovalbumin upstream promoter-transcription factors (COUP-TFs): Coming of age. Endocrinol. Rev. 18: 229-240.

Ulrich, D. 1995. Color atlas of embryology. Thieme Medical Publishers, New York, NY.

van der Wees, J., P.J. Matharu, K. de Roos, O.H. Destree, S.F. Godsave, A.J. Durston, and G.E. Sweeney. 1996. Developmental expression and differential regulation by retinoic acid of Xenopus COUP-TF-A and COUP-TF-B. Mech. Dev. 54: 173-184.

Vikkula, M., L.M. Boon, K.L. Carraway, 3rd, J.T. Calvert, A.J. Diamonti, B. Goumnerov, K.A. Pasyk, D.A. Marchuk, M.L. Warman, L.C. Cantley, J.B. Mulliken, and B.R. Olsen. 1996. Vascular dysmorphogenesis caused by an activating mutation in the receptor tyrosine kinase TIE2. Cell 87: 1181-1190.

Wang, H.U., Z.F. Chen, and D.J. Anderson. 1998. Molecular distinction and angiogenic interaction between embryonic arteries and veins revealed by ephrin-B2 and its receptor EphB4. Cell 93: 741-753.

Yamaguchi, T.P., D.J. Dumont, R.A. Conlon, M.L. Breitman, and J. Rossant. 1993. flk-1, an flt-related receptor tyrosine kinase is an early marker for endothelial cell precursors. Development 118: 489-498.

Zeng, X., S.E. Wert, R. Federici, K.G. Peters, and J.A. Whitsett. 1998. VEGF enhances pulmonary vasculogenesis and disrupts lung morphogenesis in vivo. Dev. Dyn. 211: 215-227. 


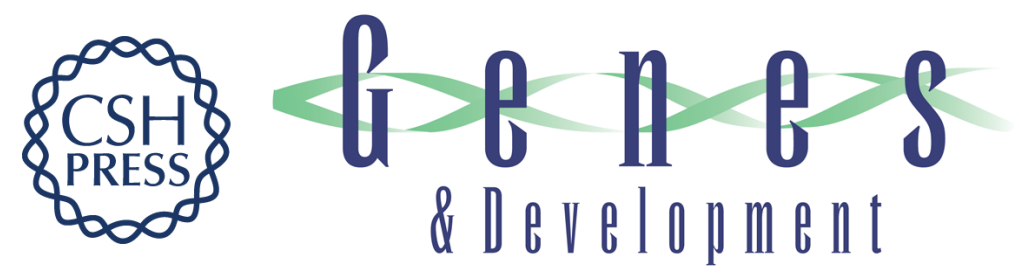

\section{The orphan nuclear receptor COUP-TFII is required for angiogenesis and heart development}

Fred A. Pereira, Yuhong Qiu, Ge Zhou, et al.

Genes Dev. 1999, 13:

References This article cites 47 articles, 17 of which can be accessed free at:

http://genesdev.cshlp.org/content/13/8/1037.full.html\#ref-list-1

License

Email Alerting
Service

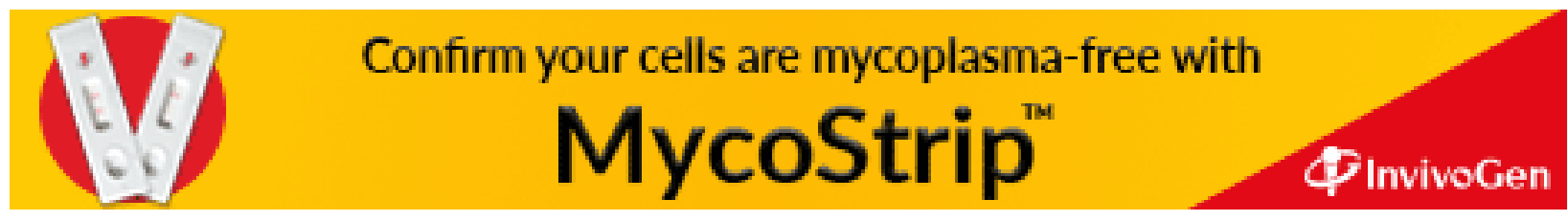

